\title{
Acyclic, Star and Oriented Colourings of Graph Subdivisions
}

\author{
David R. Wood \\ Departament de Matemàtica Aplicada II, Universitat Politècnica de Catalunya, Barcelona, Spain \\ david.woodeupc.edu
}

received August 7, 2004, revised March 30, 2005, accepted April 3, 2005.

Let $G$ be a graph with chromatic number $\chi(G)$. A vertex colouring of $G$ is acyclic if each bichromatic subgraph is a forest. A star colouring of $G$ is an acyclic colouring in which each bichromatic subgraph is a star forest. Let $\chi_{\mathrm{a}}(G)$ and $\chi_{\mathrm{s}}(G)$ denote the acyclic and star chromatic numbers of $G$. This paper investigates acyclic and star colourings of subdivisions. Let $G^{\prime}$ be the graph obtained from $G$ by subdividing each edge once. We prove that acyclic (respectively, star) colourings of $G^{\prime}$ correspond to vertex partitions of $G$ in which each subgraph has small arboricity (chromatic index). It follows that $\chi_{\mathrm{a}}\left(G^{\prime}\right), \chi_{\mathrm{s}}\left(G^{\prime}\right)$ and $\chi(G)$ are tied, in the sense that each is bounded by a function of the other. Moreover the binding functions that we establish are all tight. The oriented chromatic number $\vec{\chi}(G)$ of an (undirected) graph $G$ is the maximum, taken over all orientations $D$ of $G$, of the minimum number of colours in a vertex colouring of $D$ such that between any two colour classes, all edges have the same direction. We prove that $\vec{\chi}\left(G^{\prime}\right)=\chi(G)$ whenever $\chi(G) \geq 9$.

Keywords: graph, graph colouring, star colouring, star chromatic number, acyclic colouring, acyclic chromatic number, oriented colouring, oriented chromatic number, subdivision

AMS classification: $05 \mathrm{C} 15$ (coloring of graphs and hypergraphs)

\section{Introduction}

Let $G$ be a (finite, simple, undirected) graph with vertex set $V(G)$ and edge set $E(G)$. Let $\delta(G)$ and $\Delta(G)$ denote the minimum and maximum degrees of $G$.

A vertex partition of $G$ is a set $\left\{G_{1}, G_{2}, \ldots, G_{k}\right\}$ of induced subgraphs of $G$ such that $V(G)=\bigcup_{i=1}^{k} V\left(G_{i}\right)$ and $V\left(G_{i}\right) \cap V\left(G_{j}\right)=\emptyset$ for all distinct $i$ and $j$. A vertex $k$-colouring of $G$ is a vertex partition $\left\{G_{1}, G_{2}, \ldots, G_{k}\right\}$ in which $E\left(G_{i}\right)=\emptyset$ for all $i$. A vertex in $V\left(G_{i}\right)$ is said to be coloured $i$, and a vertex $k$-colouring can be

\footnotetext{
${ }^{\dagger}$ Supported by grant MEC SB2003-0270. Partially completed at the School of Computer Science, and the Department of Mathematics and Statistics, McGill University, Montréal, Canada. Partially completed at the Department of Applied Mathematics and the Institute for Theoretical Computer Science, Charles University, Prague, Czech Republic. Supported by project LN00A056 of the Ministry of Education of the Czech Republic, and by the European Union Research Training Network COMBSTRU (Combinatorial Structure of Intractable Problems).
}

1365-8050 (c) 2005 Discrete Mathematics and Theoretical Computer Science (DMTCS), Nancy, France 
viewed as a function that assigns one of $k$ colours to every vertex of $G$ such that adjacent vertices receive distinct colours. The chromatic number $\chi(G)$ is the minimum $k$ such that $G$ has a vertex $k$-colouring.

An edge partition of $G$ is a set $\left\{G_{1}, G_{2}, \ldots, G_{k}\right\}$ of subgraphs of $G$ such that $E(G)=\bigcup_{i=1}^{k} E\left(G_{i}\right)$ and $E\left(G_{i}\right) \cap E\left(G_{j}\right)=\emptyset$ for all distinct $i$ and $j$. An edge $k$-colouring of $G$ is an edge partition $\left\{G_{1}, G_{2}, \ldots, G_{k}\right\}$ of $G$ in which each $G_{i}$ is a matching. An edge in $E\left(G_{i}\right)$ is said to be coloured $i$, and an edge $k$-colouring can be viewed as a function that assigns one of $k$ colours to every edge of $G$ such that pairs of edges with a common endpoint receive distinct colours. The chromatic index $\chi^{\prime}(G)$ is the minimum $k$ such that $G$ has an edge $k$-colouring.

We will mainly be concerned with vertex colourings. Henceforth a colouring will mean a vertex colouring.

A colouring of $G$ is acyclic if every cycle receives at least three colours; that is, every bichromatic subgraph is a forest. The acyclic chromatic number $\chi_{\mathrm{a}}(G)$ is the minimum number of colours in an acyclic colouring of $G$. An acyclic colouring is a star colouring if every 4-vertex path receives at least three colours; that is, every bichromatic subgraph is a union of disjoint stars. The star chromatic number $\chi_{\mathrm{s}}(G)$ is the minimum number of colours in a star colouring of $G$. By definition every graph $G$ satisfies

$$
\chi_{\mathrm{a}}(G) \leq \chi_{\mathrm{s}}(G) .
$$

It is folklore that $\chi_{\mathrm{s}}(G) \leq \chi_{\mathrm{a}}(G) \cdot 2^{\chi_{\mathrm{a}}(G)-1}$ (see [27, 31]). Albertson et al. [3] recently improved this bound to $\chi_{\mathrm{s}}(G) \leq \chi_{\mathrm{a}}(G)\left(2 \chi_{\mathrm{a}}(G)-1\right)$. A general result by Nešetřil and Ossona de Mendez [44] states that $\chi_{\mathrm{s}}(G)$ (and hence $\chi_{\mathrm{a}}(G)$ ) is at most a quadratic function of the maximum chromatic number of a minor of $G$. Other references on acyclic and star colourings include [1, 2, 4, 5, 11, 13, 16, 17, 18, 21, 25, 26, 27, 29, 33, 34, 35, 40].

A directed graph obtained from a graph $G$ by giving each edge one of the two possible orientations is called an orientation of $G$. The arc set of an orientation $D$ is denoted by $A(D)$. A colouring of $D$ is oriented if between every pair of colour classes, all edges have the same direction. The oriented chromatic number $\vec{\chi}(D)$ is the minimum number of colours in an oriented colouring of $D$. A tournament is an orientation of a complete graph. Observe that $\vec{\chi}(D) \leq k$ if and only if there is a homomorphism $\phi$ from $D$ to a $k$-vertex tournament $H$; that is, for every arc $v w \in A(D)$, the image $\phi(v) \phi(w) \in A(H)$.

The oriented chromatic number of an (undirected) graph $G$, denoted by $\vec{\chi}(G)$, is the maximum of $\vec{\chi}(D)$, taken over all orientations $D$ of $G$. Oriented chromatic number is bounded by acyclic chromatic number. In particular, Raspaud and Sopena [48] proved that $\vec{\chi}(G) \leq \chi_{\mathrm{a}}(G) \cdot 2^{\chi_{\mathrm{a}}(G)-1}$. Other reference on oriented chromatic number include [12, 14, 15, 28, 32, 34, 45, 46, 47, 48, 50, 51, 52].

A subdivision of a graph $G$ is a graph obtained from $G$ by replacing each edge by an internally disjoint path of at least one edge. The vertices of a subdivision of $G$ corresponding to vertices of $G$ are said to be original vertices. The remaining vertices are called division vertices. The subdivision of $G$ obtained by replacing each edge $v w$ by a 3 -vertex path $(v, x, w)$ is denoted by $G^{\prime}$. Clearly $\chi\left(G^{\prime}\right) \leq 2$ for every graph $G$.

\subsection{Results}

The star / acyclic / oriented chromatic numbers of $G^{\prime}$ are the main topics of this paper. Our results on these topics are respectively presented in Sections 3,4 and 5 . We show that star (respectively, acyclic) colourings of $G^{\prime}$ correspond to vertex partitions of $G$ in which each subgraph has small chromatic index (arboricity). It follows that $\chi_{\mathrm{s}}\left(G^{\prime}\right), \chi_{\mathrm{a}}\left(G^{\prime}\right)$ and $\chi(G)$ are tied, in the sense that each is bounded by a function of the other. Moreover the binding functions that we establish are all tight. We start in Section 2 
with a general discussion of 'partitionable' parameters that may be of independent interest. In Section 5 we prove that $\vec{\chi}\left(G^{\prime}\right)$ is strongly tied to $\chi(G)$. In particular, $\vec{\chi}\left(G^{\prime}\right)=\chi(G)$ whenever $\chi(G) \geq 9$. Finally in Section 6 , we study the acyclic and star chromatic numbers of subdivisions in which each edge is replaced by a path of at least four vertices. We prove that such subdivisions have bounded star / acyclic / oriented chromatic numbers. A theme of this paper is that questions about graph colourings and partitions can be expressed in terms of colourings of subdivisions. Another example is that the total chromatic number of $G$ equals the chromatic number of the square of $G^{\prime}$.

\section{Partitionable Parameters}

The following result by Lovász [38], which will be used in Section 3, says that the maximum degree is a 'partitionable' parameter; see [8, 9, 19, 22, 30, 36, 42] for related work.

Lemma 1 ([38]). Let $G$ be a graph. Let $d_{1}, d_{2}, \ldots, d_{k}$ be non-negative integers such that $\sum_{i=1}^{k} d_{i}=\Delta(G)-$ $k+1$. Then $G$ has a vertex partition $\left\{G_{1}, G_{2}, \ldots, G_{k}\right\}$ in which $\Delta\left(G_{i}\right) \leq d_{i}$ for all $i$.

A graph is chordal if it contains no induced cycle on at least four vertices. The treewidth $\operatorname{tw}(G)$ is the minimum $k$ such that the graph $G$ is a subgraph of a chordal graph with no $(k+2)$-clique. The following result by Ding et al. [23] says that treewidth is partitionable.

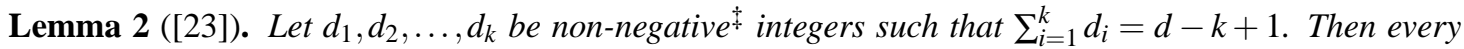
graph $G$ with treewidth $\operatorname{tw}(G) \leq d$ has a vertex partition $\left\{G_{1}, G_{2}, \ldots, G_{k}\right\}$ in which each $G_{i}$ has treewidth $\operatorname{tw}\left(G_{i}\right) \leq d_{i}$.

The degeneracy of $G$ is defined to be

$$
\mathrm{d}(G)=\max _{H \subseteq G} \delta(H) .
$$

A graph with degeneracy at most $d$ is $d$-degenerate. The following result due to Mihók [39] says that degeneracy is partitionable. We include the proof (which was discovered independently) for completeness.

Theorem 1 ([39]). Let $d_{1}, d_{2}, \ldots, d_{k}$ be non-negative integers such that $\sum_{i=1}^{k} d_{i}=d-k+1$. Then every $d$-degenerate graph $G$ has a vertex partition $\left\{G_{1}, G_{2}, \ldots, G_{k}\right\}$ in which each $G_{i}$ is $d_{i}$-degenerate.

Proof. We proceed by induction on $|V(G)|$. The result is trivial if $|V(G)|=1$. By definition, $G$ has a vertex $v$ of degree at most $d$, and $G \backslash v$ is also $d$-degenerate. By induction, $G \backslash v$ has a vertex partition $\left\{G_{1}, G_{2}, \ldots, G_{k}\right\}$ in which each $G_{i}$ is $d_{i}$-degenerate. There is some $i$ such that $G_{i}$ contains at most $d_{i}$ neighbours of $v$, as otherwise $v$ has degree at least $\sum_{i=1}^{k}\left(d_{i}+1\right)=d+1$. Let $H$ be the subgraph of $G$ induced by $V\left(G_{i}\right) \cup\{v\}$. It follows that $H$ is also $d_{i}$-degenerate (see [37, 41] for example). Thus $\left\{G_{1}, \ldots, G_{i-1}, H, G_{i+1}, \ldots, G_{k}\right\}$ is the desired vertex partition of $G$.

It is easily seen that Theorem 1 is best possible for the complete graph $K_{n}$ with $n \equiv 0(\bmod k(k+1))$, and $d_{i}=d_{j}$ for all $1 \leq i<j \leq k$.

For planar graphs, which are 5-degenerate, stronger results than Theorem 1 are possible. The 4colour theorem [49] states that every planar graph has a vertex partition into four 0 -degenerate subgraphs. Strengthening the 5-colour theorem, Thomassen [53] proved that every planar graph has a vertex partition

Ding et al. [23] state Lemma 2 for positive integers $d_{1}, d_{2}, \ldots, d_{k}$. It is easily seen that the proof is still valid if some $d_{i}=0$. A graph has treewidth 0 if and only if it has no edges. 
into a 2-degenerate subgraph and a 1-degenerate subgraph (a forest), and Thomassen [54] proved that every planar graph has a vertex partition into a 3-degenerate subgraph and a 0-degenerate subgraph.

The arboricity a $(G)$ is the minimum $k$ such that the graph $G$ has an edge partition $\left\{G_{1}, G_{2}, \ldots, G_{k}\right\}$ in which each $G_{i}$ is a forest. Nash-Williams [43] proved that

$$
\mathrm{a}(G)=\max _{H \subseteq G}\left\lceil\frac{|E(H)|}{|V(H)|-1}\right\rceil .
$$

It is well known that (see [56] for example)

$$
\mathrm{a}(G) \leq \mathrm{d}(G) \leq 2 \mathrm{a}(G)-1
$$

and

$$
\chi(G) \leq \mathrm{d}(G)+1 \leq 2 \mathrm{a}(G) .
$$

To what extent arboricity is a partitionable parameter will be important in Section 4 Theorem 1 and (3) imply:

Corollary 1. Let $G$ be a graph with degeneracy $\mathrm{d}(G) \leq d$ (which is implied if $G$ has arboricity $\mathrm{a}(G) \leq$ $\left.\frac{1}{2}(d+1)\right)$. Let $d_{1}, d_{2}, \ldots, d_{k}$ be non-negative integers such that $\sum_{i=1}^{k} d_{i}=d-k+1$. Then $G$ has a vertex partition $\left\{G_{1}, G_{2}, \ldots, G_{k}\right\}$ in which each $G_{i}$ has arboricity a $\left(G_{i}\right) \leq d_{i}$.

Corollary 2. Let $G$ be a graph with arboricity $\mathrm{a}(G) \leq d$. Let $d_{1}, d_{2}, \ldots, d_{k}$ be non-negative integers such that $\sum_{i=1}^{k} d_{i}=2 d-k$. Then $G$ has a vertex partition $\left\{G_{1}, G_{2}, \ldots, G_{k}\right\}$ in which each $G_{i}$ has arboricity $\mathrm{a}\left(G_{i}\right) \leq d_{i}$.

\section{Star Colourings of $G^{\prime}$}

In this section we study the star chromatic number of $G^{\prime}$. First we give a simple upper bound on $\chi_{\mathrm{s}}\left(G^{\prime}\right)$ in terms of $\chi(G)$.

Lemma 3. For every graph $G, \chi_{\mathrm{s}}\left(G^{\prime}\right) \leq \max \{\chi(G), 3\}$.

Proof. Consider a colouring of $G$ with $\chi(G)$ colours. Define a colouring of $G^{\prime}$ in which each original vertex inherits its colour from $G$. If $\chi(G) \leq 2$ then let all the division vertices receive one new colour. Otherwise (if $\chi(G) \geq 3$ ), for each division vertex, choose one of the $\chi(G)$ colours different from the two colours assigned to its two neighbours. A 4-vertex path in $G^{\prime}$ contains a trichromatic path $(v, x, w)$, where $x$ is the division vertex of the edge $v w$. Thus $G^{\prime}$ has a star colouring with $\max \{\chi(G), 3\}$ colours.

In Lemma 3 , the original vertices of $G^{\prime}$ inherit their colour from a colouring of $G$. At the other extreme, the original vertices of $G^{\prime}$ are monochromatic.

Lemma 4. For every graph $G$, the minimum number of colours in a star colouring of $G^{\prime}$ in which the original vertices are monochromatic is $\chi^{\prime}(G)+1$.

Proof. Given an edge colouring of $G$, transfer the colour from each edge to the corresponding division vertex, and colour all of the original vertices with a new colour. Let $P=(v, x, w, y)$ be a 4-vertex path of $G^{\prime}$. Without loss of generality, $x$ is the division vertex of the edge $v w$, and $y$ is the division vertex of some edge $w u$. In the edge colouring, $v w$ and $w u$ receive distinct colours. Hence $x$ and $y$ receive distinct colours, and 
$P$ is not bichromatic. Thus $G^{\prime}$ has a star colouring with $\chi^{\prime}(G)+1$ colours in which the original vertices are monochromatic.

Consider a star colouring of $G^{\prime}$ with $k$ colours in which the original vertices are monochromatic. No division vertex can receive this colour, otherwise it is not a colouring. For all pairs of edges of $G$ with an endpoint in common, the corresponding division vertices receive distinct colours, as otherwise there is a bichromatic 5-vertex path in $G^{\prime}$. Transferring the colour from each division vertex of $G^{\prime}$ to the corresponding edge of $G$, we obtain an edge $(k-1)$-colouring of $G$.

Theorem 2. For every graph $G$, the star chromatic number of $G^{\prime}$ satisfies:

$$
\sqrt{\chi(G)} \leq \chi_{\mathrm{s}}\left(G^{\prime}\right) \leq \max \{\chi(G), 3\}
$$

Proof. The upper bound is Lemma 3 . Let $\phi$ be a star $k$-colouring of $G^{\prime}$, where $k=\chi_{\mathrm{s}}\left(G^{\prime}\right)$. Let $H$ be the spanning subgraph of $G$ with edge set $E(H)=\{v w \in E(G): \phi(v)=\phi(w)\}$. Then every connected component of $H$ is monochromatic under $\phi$. By Lemma $4, \chi^{\prime}(H) \leq k-1$. Hence $\Delta(H) \leq k-1$, and thus $\chi(H) \leq k$ by Brooks' Theorem [20]. Let $\varphi$ be a vertex $k$-colouring of $H$. Now colour each vertex $v \in V(G)$ by the pair $(\phi(v), \varphi(v))$. Consider an edge $v w \in E(G)$. If $v w \in E(H)$ then $\varphi(v) \neq \varphi(w)$. If $v w \notin E(H)$ then $\phi(v) \neq \phi(w)$. Thus we have a $k^{2}$-colouring of $G$, and $\chi(G) \leq \chi_{\mathrm{s}}(G)^{2}$.

We now take an approach that is somewhere between the extremes of Lemmata 3 and 4.

Lemma 5. Let $G$ be a graph, and let $k \geq 1$ and $d \geq 0$ be integers. Suppose that $G$ has a vertex partition $\left\{G_{1}, G_{2}, \ldots, G_{k}\right\}$ in which $\chi^{\prime}\left(G_{i}\right) \leq d$ for all $1 \leq i \leq k$. Then $\chi_{\mathrm{s}}\left(G^{\prime}\right) \leq \max \{k+1, d+2\}$.

Proof. Let $m=\max \{k, d+1\}$ and $[m]=\{0,1, \ldots, m-1\}$. For each vertex $v \in V\left(G_{i}\right)$, let $\phi(v)=i-1$. Thus $\phi(v) \in[m]$. For $1 \leq i \leq k$, let $\lambda_{i}$ be an edge $d$-colouring of $G_{i}$, where $1 \leq \lambda_{i}(v w) \leq d$. Consider an edge $v w$ of $G$ whose division vertex in $G^{\prime}$ is $x$. First suppose that $\phi(v)=\phi(w)=i$. Let $\phi(x)=(i+$ $\left.\lambda_{i}(v w)\right) \bmod m$. Since $i \in[m]$ and $m>d \geq \lambda_{i}(v w) \geq 1, \phi(x) \in[m] \backslash\{i\}$. If $\phi(v) \neq \phi(w)$, then let $\phi(x)=m$. In both cases, $x$ is coloured differently from both of its neighbours. Hence $\phi$ is a colouring of $G^{\prime}$. Suppose that $\phi$ is not a star colouring. That is, there is a path $P=(v, x, w, y)$ in $G^{\prime}$, and $\phi(v)=\phi(w) \neq \phi(x)=\phi(y)$. Without loss of generality, $x$ is the division vertex of the edge $v w$, and $y$ is the division vertex of some edge $w u$. First suppose that $\phi(v)=\phi(w)=\phi(u)$. Then $v w$ and $w u$ are in some $G_{i}$. Hence the edge colours of $v w$ and $w u$ are distinct, and $\phi(x) \neq \phi(y)$, a contradiction. If $\phi(v)=\phi(w) \neq \phi(u)$ then $\phi(x) \leq m-1$ and $\phi(y)=m$, a contradiction. Therefore $\phi$ is a star colouring of $G^{\prime}$ with $m+1=\max \{k+1, d+2\}$ colours.

Converse to Lemma 5, we have the following.

Lemma 6. For every graph $G$, if $\chi_{\mathrm{s}}\left(G^{\prime}\right) \leq k$ then $G$ has a vertex partition $\left\{G_{1}, G_{2}, \ldots, G_{k}\right\}$ in which each $G_{i}$ has chromatic index $\chi^{\prime}\left(G_{i}\right) \leq k-1$.

Proof. Let $\phi$ be a star $k$-colouring of $G^{\prime}$. Let $\left\{G_{1}, G_{2}, \ldots, G_{k}\right\}$ be the vertex partition of $G$, where $V\left(G_{i}\right)=$ $\{v \in V(G): \phi(v)=i\}$. By Lemma $4, \chi^{\prime}\left(G_{i}\right) \leq k-1$ for all $i$.

Theorem 3. For every graph $G, \chi_{\mathrm{s}}\left(G^{\prime}\right) \leq \sqrt{\Delta(G)}+3$. 
Proof. Let $\Delta=\Delta(G)$ and $k=\lceil\sqrt{\Delta}\rceil$. Let $d_{1}, d_{2}, \ldots, d_{k} \in\{\lfloor(\Delta-k+1) / k\rfloor,\lceil(\Delta-k+1) / k\rceil\}$ such that $\sum_{i=1}^{k} d_{i}=\Delta-k+1$. By Lemma 1 , $G$ has a vertex partition $\left\{G_{1}, G_{2}, \ldots, G_{k}\right\}$ in which $\Delta\left(G_{i}\right) \leq d_{i} \leq$ $\lceil(\Delta-k+1) / k\rceil \leq \Delta / k \leq \sqrt{\Delta}$ for all $i$. By Vizing's Theorem [55], $\chi^{\prime}\left(G_{i}\right) \leq \sqrt{\Delta}+1$. By Lemma 5 . $\chi_{\mathbf{s}}\left(G^{\prime}\right) \leq \max \{\lceil\sqrt{\Delta}\rceil+1, \sqrt{\Delta}+3\} \leq \sqrt{\Delta}+3$.

The following example shows that, up to the additive constant, the lower bound in Theorem 2 and the upper bound in Theorem 3 are tight.

Example 1. For all $n \geq 1, \sqrt{n} \leq \chi_{\mathbf{s}}\left(K_{n}^{\prime}\right) \leq \sqrt{n-1}+3$.

We now prove that the upper bound in Theorem 2 is tight. Let $K\left(n_{1}, n_{2}, \ldots, n_{k}\right)$ denote the complete $k$-partite graph with $n_{i}$ vertices in the $i$-th colour class.

Example 2. For all $k \geq 3$ and $n \geq k-1$, the complete $k$-partite graph $G=K(n, n, \ldots, n)$ satisfies $\chi_{\mathbf{s}}\left(G^{\prime}\right)=$ $k(=\chi(G))$.

Proof. That $\chi_{\mathrm{s}}\left(G^{\prime}\right) \leq k$ follows from Lemma 3 . Suppose on the contrary, that $\chi_{\mathrm{s}}\left(G^{\prime}\right) \leq k-1$. By Lemma 6 Gas a vertex partition $\left\{G_{1}, G_{2}, \ldots, G_{k-1}\right\}$ in which $\chi^{\prime}\left(G_{i}\right) \leq k-2$ for all $i$, which implies that $\Delta\left(G_{i}\right) \leq k-2$. For some $1 \leq i \leq k-1,\left|V\left(G_{i}\right)\right| \geq|V(G)| /(k-1)=k n /(k-1)$. For some $1 \leq j \leq k$, the number of vertices in $V\left(G_{i}\right)$ that are in the $j$-th colour class of $G$ is at most $\left|V\left(G_{i}\right)\right| / k$. Let $v$ be such a vertex. Vertices in distinct colour classes of $G$ are adjacent. Thus $v$ is adjacent to at least $\left|V\left(G_{i}\right)\right|-\left|V\left(G_{i}\right)\right| / k$ vertices in $G_{i}$. That is, $\Delta\left(G_{i}\right) \geq(k-1)\left|V\left(G_{i}\right)\right| / k \geq n$. Thus we obtain the desired contradiction for $n \geq k-1$.

\section{Acyclic Colourings of $G^{\prime}$}

In this section we study the acyclic chromatic number of $G^{\prime}$. The results are analogous to those for the star chromatic number in Section 3, with arboricity playing the same role as chromatic index. We start with an analogue of Lemma 4

Lemma 7. For every graph $G$, the minimum number of colours in an acyclic colouring of $G^{\prime}$ in which the original vertices are monochromatic is $\mathrm{a}(G)+1$.

Proof. Suppose we have an acyclic $(k+1)$-colouring of $G^{\prime}$ in which the original vertices are monochromatic. Then no division vertex receives the same colour as the original vertices. The edge partition of $G$ defined with respect to the colour of the corresponding division vertex consists of $k$ acyclic subgraphs, and $\mathrm{a}(G) \leq k$. Conversely, given an edge partition $\left\{G_{1}, G_{2}, \ldots, G_{k}\right\}$ of $G$ into forests, let $i$ be the colour of each division vertex of an edge in $G_{i}$, and colour each original vertex 0 . We obtain an acyclic $(k+1)$-colouring of $G^{\prime}$ in which the original vertices are monochromatic.

Lemma 8. Let $d \geq 0$ and $k \geq 1$ be integers. If a graph $G$ has a vertex partition $\left\{G_{1}, G_{2}, \ldots, G_{k}\right\}$ in which each $G_{i}$ has arboricity $\mathrm{a}\left(G_{i}\right) \leq d$, then $G^{\prime}$ has acyclic chromatic number $\chi_{\mathrm{a}}\left(G^{\prime}\right) \leq \max \{k, d+1,3\}$.

Proof. For each vertex $v \in V\left(G_{i}\right)$, let $\phi(v)=i-1$. Let $m=\max \{k, d+1,3\}$ and $[m]=\{0,1, \ldots, m-1\}$. Thus $\phi(v) \in[m]$. For $1 \leq i \leq k$, let $\left\{G_{i, 1}, G_{i, 2}, \ldots, G_{i, d}\right\}$ be an edge partition of $G_{i}$ into forests. Consider an edge $v w$ of $G$ whose division vertex in $G^{\prime}$ is $x$. First suppose that $\phi(v)=\phi(w)=i$. Let $\phi(x)=$ $(i+j) \bmod m$, where $v w \in E\left(G_{i, j}\right)$. Since $i \in[m]$ and $m>d \geq j \geq 1, \phi(x) \in[m] \backslash\{i\}$. Now suppose that 
$\phi(v) \neq \phi(w)$. Choose $\phi(x) \in[m] \backslash\{\phi(v), \phi(w)\}$. Since $m \geq 3$ there is such a colour. In both cases, $x$ is coloured differently from both of its neighbours. Hence $\phi$ is a colouring of $G^{\prime}$.

Suppose on the contrary that under $\phi$, there is a bichromatic cycle $C$ in $G^{\prime}$. Then for some $t, C=$ $\left(v_{0}, x_{0}, v_{1}, x_{1}, \ldots, v_{t-1}, x_{t-1}\right)$, where each $v_{\alpha}$ is an original vertex, each $x_{\alpha}$ is the division vertex of $v_{\alpha} v_{\alpha+1}$ (modulo $t$ ), and $\phi\left(v_{\alpha}\right)=\phi\left(v_{\beta}\right)$ and $\phi\left(x_{\alpha}\right)=\phi\left(x_{\beta}\right)$ for all $\alpha$ and $\beta$. Thus by the definition of $\phi$, for some $1 \leq i \leq k$, every vertex $v_{\alpha} \in V\left(G_{i}\right)$, which implies that for some $1 \leq j \leq d$, every edge $v_{\alpha} v_{\alpha+1} \in E_{j}^{i}$. Hence $G_{i, j}$ contains a cycle, a contradiction. Thus $\phi$ is an acyclic $m$-colouring of $G^{\prime}$.

Theorem 4. Let $G$ be a graph and $k \geq 2$ be an integer. Then $\chi_{\mathrm{a}}\left(G^{\prime}\right) \leq k$ if and only if $G$ has a vertex partition $\left\{G_{1}, G_{2}, \ldots, G_{k}\right\}$ in which each $G_{i}$ has arboricity $\mathrm{a}\left(G_{i}\right) \leq k-1$.

Proof. $(\Leftarrow)$ This is Lemma 8 with $d=k-1$.

$(\Rightarrow)$ Consider the vertex partition of $G$ defined by an acyclic $k$-colouring of $G^{\prime}$ (restricted to $G$ ). By Lemma 7, each subgraph has arboricity at most $k-1$.

Theorem 5. For every graph $G$ with degeneracy $\mathrm{d}(G) \leq d$ (which is implied if $G$ has arboricity $\mathrm{a}(G) \leq$ $\left.\frac{1}{2}(d+1)\right), \chi_{\mathrm{a}}\left(G^{\prime}\right) \leq \max \{\sqrt{d}+1,3\}$.

Proof. Let $k=\lceil\sqrt{d}\rceil$. Let $d_{1}, d_{2}, \ldots, d_{k} \in\{\lfloor(d-k+1) / k\rfloor,\lceil(d-k+1) / k\rceil\}$ such that $\sum_{i=1}^{k} d_{i}=d-k+1$. By Corollary 1, $G$ has a vertex partition $\left\{G_{1}, G_{2}, \ldots, G_{k}\right\}$ in which a $\left(G_{i}\right) \leq d_{i} \leq\lceil(d-k+1) / k\rceil \leq d / k \leq$ $\sqrt{d}$ for all $i$. By Lemma $8, \chi_{\mathrm{a}}\left(G^{\prime}\right) \leq \max \{\lceil\sqrt{d}\rceil, \sqrt{d}+1,3\}=\max \{\sqrt{d}+1,3\}$.

Theorem 6. For every graph $G$, if $\chi_{\mathrm{a}}\left(G^{\prime}\right) \leq k$ then $\chi(G) \leq 2 k(k-1)$.

Proof. Let $\phi$ be an acyclic $k$-colouring of $G^{\prime}$. Let $H$ be the spanning subgraph of $G$ with edge set $E(H)=$ $\{v w \in E(G): \phi(v)=\phi(w)\}$. Then every connected component of $H$ is monochromatic under $\phi$. By Lemma 7, $H$ has arboricity at most $k-1$. By (4), $H$ has a vertex $2(k-1)$-colouring $\varphi$. Now colour each vertex $v \in V(G)$ by the pair $(\phi(v), \varphi(v))$. Consider an edge $v w \in E(G)$. If $v w \in E(H)$ then $\varphi(v) \neq \varphi(w)$. If $v w \notin E(H)$ then $\phi(v) \neq \phi(w)$. Thus we have a $2 k(k-1)$-colouring of $G$.

Lemma 3 and Theorem 6 and (1) imply that $\chi_{\mathrm{a}}\left(G^{\prime}\right)$ is tied to $\chi(G)$.

Corollary 3. For every graph $G$, the acyclic chromatic number of $G^{\prime}$ satisfies:

$$
\sqrt{\frac{1}{2} \chi(G)}<\chi_{\mathrm{a}}\left(G^{\prime}\right) \leq \max \{\chi(G), 3\}
$$

The following example shows that the lower bound in Corollary 3 is tight up to an additive constant.

Example 3. For all $n, \sqrt{n / 2}<\chi_{\mathrm{a}}\left(K_{n}^{\prime}\right)<\sqrt{n / 2}+\frac{5}{2}$

Proof. The lower bound follows from Corollary 3 . Now we prove the upper bound. Observe that a $\left(K_{n}\right)=$ $\lceil n / 2\rceil$ by (2). Let $k=\lceil\sqrt{n / 2}\rceil$. Let $\left\{G_{1}, G_{2}, \ldots, G_{k}\right\}$ be a vertex partition of $K_{n}$, in which $\left|V\left(G_{i}\right)\right| \in$ $\{\lfloor n / k\rfloor,\lceil n / k\rceil\}$ for all $i$. By the above observation,

$$
\mathrm{a}\left(G_{i}\right) \leq\left\lceil\frac{1}{2}\lceil n / k\rceil\right\rceil \leq\left\lceil\frac{1}{2}\lceil n / \sqrt{n / 2}\rceil\right\rceil=\left\lceil\frac{1}{2}\lceil\sqrt{2 n}\rceil\right\rceil<\left\lceil\frac{1}{2}(\sqrt{2 n}+1)\right\rceil=\left\lceil\sqrt{n / 2}+\frac{1}{2}\right\rceil .
$$


By Lemma 8, $K_{n}^{\prime}$ has acyclic chromatic number

$$
\chi_{\mathrm{a}}\left(K_{n}^{\prime}\right) \leq \max \left\{\lceil\sqrt{n / 2}\rceil,\left\lceil\sqrt{n / 2}+\frac{1}{2}\right\rceil+1,3\right\}<\sqrt{n / 2}+\frac{5}{2} .
$$

We now prove that the above upper bound in Corollary 3 is tight.

Example 4. For all $k \geq 3$ and $n>n(k)$, the complete $k$-partite graph $G=K(n, n, \ldots, n)$ satisfies $\chi_{\mathrm{a}}\left(G^{\prime}\right)=$ $k(=\chi(G))$.

Proof. That $\chi_{\mathrm{a}}\left(G^{\prime}\right) \leq k$ follows from Corollary 3. Suppose on the contrary, that $\chi_{\mathrm{a}}\left(G^{\prime}\right) \leq k-1$. By Theorem 4, $G$ has a vertex partition $\left\{G_{1}, G_{2}, \ldots, G_{k-1}\right\}$ in which each $G_{i}$ has arboricity a $\left(G_{i}\right) \leq k-2$. For some $1 \leq i \leq k-1,\left|V\left(G_{i}\right)\right| \geq|V(G)| /(k-1)=k n /(k-1)$. It is easily seen that any complete $k$-partite graph $H$ on $m$ vertices has arboricity at least the arboricity of the complete $k$-partite graph $K(1,1, \ldots, 1, m-(k-1))$. This graph has $(k-1)(m-(k-1))$ edges. By (2),

$$
\mathrm{a}(H) \geq \frac{(k-1)(m-(k-1))}{m-1}=k-1-\frac{(k-1)(k-2)}{m-1} .
$$

Applying this observation with $H=G_{i}$ and $m \geq k n /(k-1)$, we have

$$
\mathrm{a}\left(G_{i}\right) \geq k-1-\frac{(k-1)(k-2)}{k n /(k-1)-1} .
$$

Since $\mathrm{a}\left(G_{i}\right) \leq k-2$, it follows that we obtain a contradiction for $n>n(k)=\left((k-1)^{2}(k-2)+(k-\right.$ $1)) / k$.

\section{Oriented Colourings of $G^{\prime}$}

We now relate the oriented chromatic number of $G^{\prime}$ to the chromatic number of $G$.

Theorem 7. For every graph $G$, the oriented chromatic number of $G^{\prime}$ satisfies

$$
\chi(G) \leq \vec{\chi}\left(G^{\prime}\right) \leq \begin{cases}7 & \text { if } \chi(G) \leq 7 \\ 9 & \text { if } \chi(G)=8 \\ \chi(G) & \text { if } \chi(G) \geq 9\end{cases}
$$

Proof. First we prove the lower bound (which is well known). Let $D^{\prime}$ be an orientation of $G^{\prime}$ in which each division vertex has one incoming arc and one outgoing arc. Consider an edge $v w \in E(G)$ whose division vertex in $G^{\prime}$ is $x$. In any oriented colouring of $D^{\prime}, v$ and $w$ receive distinct colours, as otherwise the arcs $v x$ and $x w$ (or $x v$ and $w x$ ) are in opposite directions between the same pair of colour classes. Thus an oriented colouring of $D^{\prime}$ contains a colouring of $G$. Hence $\vec{\chi}\left(D^{\prime}\right) \geq \chi(G)$, which implies that $G^{\prime}$ has oriented chromatic number $\vec{\chi}\left(G^{\prime}\right) \geq \chi(G)$.

Now for the upper bound. A tournament $H$ is $k$-existentially closed if for every $k$-element set of vertices $S \subseteq V(H)$ and for every (possibly empty) $T \subseteq S$, there is a vertex $z \in V(H) \backslash(S \cup T)$ such that $v z \in A(H)$ for every vertex $v \in S \backslash T$, and $z w \in A(H)$ for every vertex $w \in T$. Almost every sufficiently large tournament 
is $n$-existentially closed (see [7, 10, 24]). Note that a tournament $H$ is 2-existentially closed if and only if for every pair of vertices $v, w \in V(H)$, there exists four other vertices $a, b, c, d \in V(H)$ such that

$$
v a, w a, b v, b w, v c, c w, d v, w d \in A(H)
$$

Bonato and Cameron [10] proved that there is a 2-existentially closed tournament on $n$ vertices if and only if $n \geq 7$ and $n \neq 8$. Moreover, they provided explicit examples for all such $n$. These examples are based on the so-called Paley tournament, which for prime $n \equiv 3(\bmod 4)$, has vertex set $\{0,1, \ldots, n-1\}$, and $i j$ is an arc whenever $j-i$ is a quadratic residue modulo $p$. Note that Ananchuen [6] also proved that a sufficiently large Paley tournament is $k$-existentially closed, and Ochem [47] recently used Paley tournaments in results about oriented colourings.

Let $n$ be the claimed upper bound on $\vec{\chi}\left(G^{\prime}\right)$. Then $n \geq 7$ and $n \neq 8$. Thus there is a 2-existentially closed tournament $H$ on $n$ vertices. Let $D^{\prime}$ be an orientation of $G^{\prime}$. Note that $n \geq \chi(G)$. Fix a vertex $n$-colouring of $G$. Let $\phi$ be a function from the original vertices of $G^{\prime}$ to $V(H)$, such that $\phi(v)=\phi(w)$ if and only if $v$ and $w$ receive the same colour in the colouring of $G$. Consider a division vertex $x$ of an edge $v w \in E(G)$. By (5), there are four other vertices $a, b, c, d \in V(H)$ such that

$$
\phi(v) a, \phi(w) a, b \phi(v), b \phi(w), \phi(v) c, c \phi(w), d \phi(v), \phi(w) d \in A(H) .
$$

Define

$$
\phi(x)=\left\{\begin{array}{ll}
a & \text { if } v x, w x \in A\left(D^{\prime}\right) \\
b & \text { if } x v, x w \in A\left(D^{\prime}\right) \\
c & \text { if } v x, x w \in A\left(D^{\prime}\right) \\
d & \text { if } x v, w x \in A\left(D^{\prime}\right)
\end{array} .\right.
$$

Clearly $\phi$ is a homomorphism from $D^{\prime}$ to $H$. Thus $\vec{\chi}\left(G^{\prime}\right) \leq n$.

\section{Large Subdivisions}

In this section we consider colourings of subdivisions other than $G^{\prime}$. First we consider acyclic colourings.

Lemma 9. Let $X$ be a subdivision of a graph $G$ in which every edge of $G$ is replaced in $X$ by a path with at least four vertices; that is, every edge is subdivided at least twice. Then $\chi_{\mathrm{a}}(X) \leq 3$.

Proof. Let $\phi(v)=2$ for every original vertex $v$ of $X$. Let $D$ be an arbitrary orientation of $G$. Consider an arc $v w \in A(D)$ that is replaced by a path $\left(v, x_{0}, x_{1}, \ldots, x_{k}, w\right)$ in $X$ (for some $\left.k \geq 1\right)$. Let $\phi\left(x_{i}\right)=i \bmod 2$. Every cycle of $X$ contains a 3 -vertex path $\left(v, x_{0}, x_{1}\right)$, which is coloured $(2,0,1)$. Thus $\phi$ is an acyclic 3-colouring of $X$.

Now we consider star colourings of subdivisions other than $G^{\prime}$.

Lemma 10. Let $X$ be a subdivision of a graph $G$ such that for every edge $v w$ of $G$, for some $k \geq 4$ with $k \neq 6$, vw is replaced by a $k$-vertex path in $X$. Then $\chi_{\mathrm{s}}(X) \leq 3$.

Proof. Colour each original vertex $\phi(v)=2$. Consider an edge $v w$ of $G$ that is replaced by the $k$-vertex path $P=\left(v, x_{0}, x_{1}, \ldots, x_{k-3}, w\right)$ in $X$. 
Case 1. $k \equiv 0(\bmod 3)$ and $k \neq 6$ : Let $\phi\left(x_{i}\right)=i \bmod 3$ for all $i, 0 \leq i \leq k-6$. Let $\phi\left(x_{k-5}\right)=2$, $\phi\left(x_{k-4}\right)=1$, and $\phi\left(x_{k-3}\right)=0$. Hence $P$ is coloured $(2,012,012, \ldots, 012,0,210,2)$.

Case 2. $k \equiv 1(\bmod 3)$ : Let $\phi\left(x_{i}\right)=i \bmod 3$ for all $i, 0 \leq i \leq k-5$. Let $\phi\left(x_{k-4}\right)=1$ and $\phi\left(x_{k-3}\right)=0$. Hence $P$ is coloured $(2,012,012, \ldots, 012,10,2)$.

Case 3. $k \equiv 2(\bmod 3)$ : Let $\phi\left(x_{i}\right)=i \bmod 3$ for all $i, 0 \leq i \leq k-4$. Let $\phi\left(x_{k-3}\right)=0$. Hence $P$ is coloured $(2,012,012, \ldots, 012,01,0,2)$.

If $Q$ is a 4-vertex path in $X$ with at least two original vertices then $Q=\left(v, x_{0}, x_{1}, w\right)$, where $Q$ replaced an edge $v w$ of $G$, and by Case 2 with $k=4, Q$ is coloured $(2,1,0,2)$, and is thus not bichromatic.

If the edge $v w$ of $G$ is replaced by the path $\left(v, x_{0}, x_{1}, \ldots, x_{k-3}, w\right)$, then the subpaths $\left(v, x_{0}, x_{1}\right)$ and $\left(w, x_{k-3}, x_{k-2}\right)$ are trichromatic. (This is not the case if $k=6$.) Thus a 4-vertex path containing exactly one original vertex is not bichromatic.

The case-analysis above shows that there is no bichromatic 4 -vertex path with no original vertex. Thus there is no bichromatic 4-vertex path in $X$. Therefore $\chi_{\mathrm{s}}(X) \leq 3$.

Lemma 11. Let $X$ be a subdivision of a graph $G$ in which every edge of $G$ is replaced in $X$ by a path with at least four vertices; that is, every edge is subdivided at least twice. Then $\chi_{\mathrm{s}}(X) \leq 4$.

Proof. In the proof of Lemma 10 , the only obstruction to $X$ having a star colouring with three colours is an edge $v w$ of $G$ that is replaced in $X$ by a 6-vertex path $P=\left(v, x_{0}, x_{1}, x_{2}, x_{3}, w\right)$. In this case we introduce a fourth colour, and $P$ can be coloured $(2,0,1,3,0,2)$.

Let $G^{\prime \prime}$ be the subdivision of a graph $G$ with every edge $v w$ of $G$ replaced by a 4-vertex path with endpoints $v$ and $w$; that is, every edge is subdivided twice. A $k$-cycle in $G$ becomes a $3 k$-cycle in $G^{\prime \prime}$. Thus $G$ is bipartite if and only if $G^{\prime \prime}$ is bipartite. If $G$ contains an odd cycle, then $\chi\left(G^{\prime \prime}\right)=\chi_{\mathrm{s}}\left(G^{\prime \prime}\right)=\chi_{\mathrm{a}}\left(G^{\prime \prime}\right)=3$. This provides an infinite family of graphs for which the chromatic number, star chromatic number and acyclic chromatic number coincide.

Finally we consider oriented colourings of subdivisions other than $G^{\prime}$.

Lemma 12. Let $X$ be a subdivision of a graph $G$ in which every edge of $G$ is replaced in $X$ by a path with at least four vertices; that is, every edge is subdivided at least twice. Then $\vec{\chi}(X) \leq 5$.

Proof. Let $H$ be the tournament with $V(H)=\{0,1,2,3,4\}$, where $i j \in A(H)$ if and only if $(j-i)$ mod $5 \in\{1,2\}$. Let $D$ be an orientation of $X$. We will construct a homomorphism $\phi$ from $D$ to $H$. First define $\phi(v)=0$ for every original vertex $v$ of $X$. Consider the path $\left(v=d_{0}, d_{1}, d_{2}, \ldots, d_{t-1}, w=d_{t}\right)$ in $X$ corresponding to an edge $v w \in E(G)$. Then $t \geq 3$. For $1 \leq i \leq t$, define $x_{i}=1$ if $d_{i-1} d_{i} \in A(D)$, and define $x_{i}=-1$ if $d_{i} d_{i-1} \in A(D)$. By Lemma 13 below, there exist $y_{1}, y_{2}, \ldots, y_{t}$ such that $y_{i} \in\{1,2\}$ and $\sum_{i=1}^{t} x_{i} y_{i} \equiv 0(\bmod 5)$. For $1 \leq i \leq t-1$, set $\phi\left(d_{i}\right)=\left(\sum_{j=1}^{i} x_{j} y_{j}\right) \bmod 5$.

Consider $1 \leq i \leq t$. We have $\phi\left(d_{i}\right)-\phi\left(d_{i-1}\right) \in\{1,2\}$ whenever $x_{i}=1$; that is, when $d_{i-1} d_{i} \in A(D)$. Similarly $\phi\left(d_{i}\right)-\phi\left(d_{i-1}\right) \in\{-1,-2\}$ whenever $x_{i}=-1$; that is, when $d_{i} d_{i-1} \in A(D)$. By the definition of $H, \phi\left(d_{i-1}\right) \phi\left(d_{i}\right) \in A(H)$ for all $1 \leq i \leq t$. Hence $\phi$ is a homomorphism from $D$ to $H$, and $\vec{\chi}(X) \leq 5$.

Lemma 13. For all integers $t \geq 3$ and $x_{1}, x_{2}, \ldots, x_{t} \in\{1,-1\}$, there exist $y_{1}, y_{2}, \ldots, y_{t}$ such that $y_{i} \in\{1,2\}$ and $\sum_{i=1}^{t} x_{i} y_{i} \equiv 0(\bmod 5)$. 
Proof. Initially set every $y_{i}=1$. If $\sum_{i=1}^{t} x_{i} y_{i} \equiv 0(\bmod 5)$, then we are done.

Now suppose that $\sum_{i=1}^{t} x_{i} y_{i} \equiv 1(\bmod 5)$. If there exists $x_{i}=-1$, then set $y_{i}=2$, and we are done. Otherwise every $x_{i}=1$. Thus $t \equiv 1(\bmod 5)$ and $t \geq 6$. Set $y_{1}=y_{2}=y_{3}=y_{4}=2$, and we are done.

Now suppose that $\sum_{i=1}^{t} x_{i} y_{i} \equiv 2(\bmod 5)$. If there exists $x_{i}=x_{j}=-1$ for some $i \neq j$, then set $y_{i}=y_{j}=2$, and we are done. If there exists $i$ such that $x_{i}=-1$ and $x_{j}=1$ for all $j \neq i$, then $t-2 \equiv 2(\bmod 5)$ and $t \geq 4$; set $y_{j}=y_{k}=y_{\ell}=2$ for some distinct $j, k, \ell \neq i$, and we are done. Otherwise every $x_{i}=1$. Thus $t \equiv 2(\bmod 5)$ and $t \geq 7$. Set $y_{1}=y_{2}=y_{3}=2$, and we are done.

The cases when $\sum_{i=1}^{t} x_{i} y_{i} \equiv 3(\bmod 5)$ and $\sum_{i=1}^{t} x_{i} y_{i} \equiv 4(\bmod 5)$ are symmetric.

\section{Acknowledgements}

Thanks to Ferran Hurtado and Prosenjit Bose for graciously hosting the author. Thanks to Pascal Ochem for a number of instructive comments. Thanks to an anonymous referee for pointing out an error in the original submission.

\section{References}

[1] Michael O. Albertson And David M. Berman. Every planar graph has an acyclic 7-coloring. Israel J. Math., 28(1-2):169-174, 1977.

[2] Michael O. Albertson And David M. Berman. An acyclic analogue to Heawood's theorem. Glasgow Math. J., 19(2):163-166, 1978.

[3] Michael O. Albertson, Glenn G. Chappell, Hal A. Kierstead, André Kündgen, AND Radhika Ramamurthi. Coloring with no 2-colored $P_{4}$ 's. Electron. J. Combin., 11 \#R26, 2004.

[4] Noga Alon, Colin McDiarmid, and Bruce Reed. Acyclic coloring of graphs. Random Structures Algorithms, 2(3):277-288, 1991.

[5] Noga Alon, Bojan Mohar, and Daniel P. Sanders. On acyclic colorings of graphs on surfaces. Israel J. Math., 94:273-283, 1996.

[6] Watcharaphong Ananchuen. Graphs with prescribed adjacency properties. Ph.D. thesis, School of Mathematics and Statistics, Curtin University of Technology, Perth, Australia, 1993.

[7] Watcharaphong ANANChUEn AND Louis CACCETTA. On tournaments with a prescribed property. Ars Combin., 36:89-96, 1993.

[8] Claudio Bernardi. On a theorem about vertex colorings of graphs. Discrete Math., 64(1):95-96, 1987.

[9] Béla Bollobás and Bennet Manvel. Optimal vertex partitions. Bull. London Math. Soc., 11(2):113-116, 1979.

[10] Anthony Bonato and Kathie Cameron. On an adjacency property of almost all tournaments. Discrete Math., to appear. 
[11] Oleg V. Borodin. On acyclic colorings of planar graphs. Discrete Math., 25(3):211-236, 1979.

[12] Oleg V. Borodin, Dmitry Fon-Der-Flaass, Alexandr V. Kostochka, André RasPAUD, AND ÉRIC SOPENA. On deeply critical oriented graphs. J. Combin. Theory Ser. B, 81(1):150$155,2001$.

[13] Oleg V. Borodin, Dmitry G. Fon-Der-Flaass, Alexandr V. KostochKa, André RasPAUD, AND ÉRIC SOPENA. Acyclic list 7-coloring of planar graphs. J. Graph Theory, 40(2):83-90, 2002.

[14] Oleg V. Borodin, Alexandr V. Kostochka, Jaroslav NeŠetřIl, André Raspaud, And ÉRIC SOPENA. On universal graphs for planar oriented graphs of a given girth. Discrete Math., 188(1-3):73-85, 1998.

[15] Oleg V. Borodin, Alexandr V. Kostochka, Jaroslav NeŠEtŘIl, André Raspaud, AND ÉRIC SOPENA. On the maximum average degree and the oriented chromatic number of a graph. Discrete Math., 206(1-3):77-89, 1999.

[16] Oleg V. Borodin, Alexandr V. Kostochka, André Raspaud, and Éric. Sopena. Acyclic $k$-strong coloring of maps on surfaces. Mat. Zametki, 67(1):36-45, 2000.

[17] Oleg V. Borodin, Alexandr V. Kostochka, André Raspaud, and Éric. Sopena. Acyclic colouring of 1-planar graphs. Discrete Appl. Math., 114(1-3):29-41, 2001.

[18] Oleg V. Borodin, Alexandr V. Kostochka, And D. R. Woodall. Acyclic colourings of planar graphs with large girth. J. London Math. Soc. (2), 60(2):344-352, 1999.

[19] MieczysŁaw Borowiecki, Izak Broere, Marietjie Frick, Peter Mihók, and Gabriel SEMAnIŠIn. A survey of hereditary properties of graphs. Discuss. Math. Graph Theory, 17(1):5-50, 1997.

[20] R. L. Brooks. On colouring the nodes of a network. Proc. Cambridge Philos. Soc., 37:194-197, 1941.

[21] M. I. BURŠTEǏn. Every 4-valent graph has an acyclic 5-coloring. Soobshch. Akad. Nauk Gruzin. SSR, 93(1):21-24, 1979.

[22] VaŠEk ChVÁtal. Remark on a paper of Lovász. Comment. Math. Univ. Carolinae, 9:47-50, 1968.

[23] Guoli Ding, Bogdan Oporowski, Daniel P. Sanders, and Dirk Vertigan. Partitioning graphs of bounded tree-width. Combinatorica, 18(1):1-12, 1998.

[24] PAul ERDős. On a problem in graph theory. Math. Gaz., 47:220-223, 1963.

[25] Guillaume Fertin, Emmanuel Godard, And André Raspaud. Minimum feedback vertex set and acyclic coloring. Inform. Process. Lett., 84(3):131-139, 2002.

[26] Guillaume Fertin, Emmanuel Godard, and André Raspaud. Acyclic and $k$-distance coloring of the grid. Inform. Process. Lett., 87(1):51-58, 2003. 
[27] Guillaume Fertin, André Raspaud, ANd Bruce Reed. On star coloring of graphs. J. Graph Theory, 47(3):163-182, 2004.

[28] Guillaume Fertin, André Raspaud, and Arup Roychowdhury. On the oriented chromatic number of grids. Inform. Process. Lett., 85(5):261-266, 2003.

[29] IOSIF FIAмČík. The acyclic chromatic class of a graph. Math. Slovaca, 28(2):139-145, 1978.

[30] LÁSZLÓ GERENCSÉr. On coloring problems. Mat. Lapok, 16:274-277, 1965.

[31] BRanko Grünbaum. Acyclic colorings of planar graphs. Israel J. Math., 14:390-408, 1973.

[32] Alexandr V. Kostochka, Tomasz Łuczak, Gábor Simonyi, and Éric Sopena. On the minimum number of edges giving maximum oriented chromatic number. In Contemporary trends in discrete mathematics, pp. 179-182. Amer. Math. Soc., 1999.

[33] Alexandr V. Kostochka And Leonid S. Mel'nikov. Note to the paper of Grünbaum on acyclic colorings. Discrete Math., 14(4):403-406, 1976.

[34] Alexandr V. Kostochka, Éric Sopena, And Xuding Zhu. Acyclic and oriented chromatic numbers of graphs. J. Graph Theory, 24(4):331-340, 1997.

[35] Alexandr V. KostočKa. Acyclic 6-coloring of planar graphs. Diskret. Analiz, 28:40-54, 1976.

[36] Jim LAWRENCE. Covering the vertex set of a graph with subgraphs of smaller degree. Discrete Math., 21(1):61-68, 1978.

[37] Don R. Lick And Arthur T. White. k-degenerate graphs. Canad. J. Math., 22:1082-1096, 1970.

[38] LÁsZLó LovÁsz. On decomposition of graphs. Studia Sci. Math. Hungar., 1:237-238, 1966.

[39] Peter Мiнóк. Minimal reducible bounds for the class of $k$-degenerate graphs. Discrete Math., 236(1-3):273-279, 2001.

[40] John Mitchem. Every planar graph has an acyclic 8-coloring. Duke Math. J., 41:177-181, 1974.

[41] John Mitchem. An extension of Brooks' theorem to $n$-degenerate graphs. Discrete Math., 17(3):291-298, 1977.

[42] N. N. Mozhan. Density and n-clique chromatic number. Issled. Operatsĭ i ASU, (37):54-59, 1991.

[43] Crispin St. J. A. Nash-Williams. Decomposition of finite graphs into forests. J. London Math. Soc., 39:12, 1964.

[44] Jaroslav NeŠEtŘil and Patrice Ossona de Mendez. Colorings and homomorphisms of minor closed classes. In Boris Aronov, Saugata Basu, János PACH, AND Micha SHARIR, eds., Discrete and Computational Geometry, The Goodman-Pollack Festschrift, vol. 25 of Algorithms and Combinatorics, pp. 651-664. Springer, 2003. 
[45] Jaroslav NeŠEtřil, André Raspaud, and Éric Sopena. Colorings and girth of oriented planar graphs. Discrete Math., 165/166:519-530, 1997.

[46] JaRoslaV NeŠEtŘil, ÉRIC Sopena, AND LaURenCe Vignal. T-preserving homomorphisms of oriented graphs. Comment. Math. Univ. Carolin., 38(1):125-136, 1997.

[47] Pascal Ochem. Oriented colorings of triangle-free planar graphs. Inform. Process. Lett., 92(2):71-76, 2004.

[48] ANDRÉ RASPAUd ANd ÉRIC SOPENA. Good and semi-strong colorings of oriented planar graphs. Inform. Process. Lett., 51(4):171-174, 1994.

[49] Neil Robertson, Daniel P. Sanders, Paul Seymour, and Robin Thomas. The fourcolour theorem. J. Combin. Theory Ser. B, 70(1):2-44, 1997.

[50] ÉRIC Sopena. The chromatic number of oriented graphs. J. Graph Theory, 25(3):191-205, 1997.

[51] ÉRIC SOPEnA. Oriented graph coloring. Discrete Math., 229(1-3):359-369, 2001.

[52] ÉRIC Sopena. There exist oriented planar graphs with oriented chromatic number at least sixteen. Inform. Process. Lett., 81(6):309-312, 2002.

[53] Carsten Thomassen. Decomposing a planar graph into degenerate graphs. J. Combin. Theory Ser. B, 65(2):305-314, 1995.

[54] Carsten Thomassen. Decomposing a planar graph into an independent set and a 3-degenerate graph. J. Combin. Theory Ser. B, 83(2):262-271, 2001.

[55] Vadim G. Vizing. On an estimate of the chromatic class of a p-graph. Diskret. Analiz No., 3:2530, 1964.

[56] Xiao Zhou and TaKao NishizeKi. Edge-coloring and $f$-coloring for various classes of graphs. J. Graph Algorithms Appl., 3(1):1-18, 1999. 